\title{
Advances in biomolecular surface meshing and its applications to mathematical modeling
}

\author{
CHEN MinXin ${ }^{1,2} \&$ LU BenZhuo ${ }^{2 *}$ \\ ${ }^{1}$ Center for System Biology, Department of Mathematics, Soochow University, Suzhou 215006, China; \\ ${ }^{2}$ State Key Laboratory of Scientific/Engineering Computing, Institute of Computational Mathematics, Academy of Mathematics and Systems Science, \\ National Center for Mathematics and Interdisciplinary Sciences, Chinese Academy of Sciences, Beijing 100190, China
}

Received November 30, 2012; accepted January 24, 2013

\begin{abstract}
In the field of molecular modeling and simulation, molecular surface meshes are necessary for many problems, such as molecular structure visualization and analysis, docking problem and implicit solvent modeling and simulation. Recently, with the developments of advanced mathematical modeling in the field of implicit solvent modeling and simulation, providing surface meshes with good qualities efficiently for large real biomolecular systems becomes an urgent issue beyond its traditional purposes for visualization and geometry analyses for molecular structure. In this review, we summarize recent works on this issue. First, various definitions of molecular surfaces and corresponding meshing methods are introduced. Second, our recent meshing tool, TMSmesh, and its performances are presented. Finally, we show the applications of the molecular surface mesh in implicit solvent modeling and simulations using boundary element method (BEM) and finite element method (FEM).
\end{abstract}

molecular surface meshing, manifold mesh, mathematical modeling, TMSmesh

Citation: Chen M X, Lu B Z. Advances in biomolecular surface meshing and its applications to mathematical modeling. Chin Sci Bull, 2013, 58: 1843-1849, doi: $10.1007 / \mathrm{s} 11434-013-5829-8$

One main goal of computational structural biology/chemistry is to understand the functions and mechanisms of biomolecular systems and their relations to molecular structures with a wide range of computational approaches. To achieve this, proper and correct representation of the interface between biomolecule and solvent, molecular surface, is a primary task. In the field of implicit solvent modeling and continuum modeling, the molecular surface is used to model the dielectric interface which is the transition from the low-dielectric solute region to the high-dielectric solvent region. It is also important and useful in materials science and surface science [1]. Meshing molecular surface is a prerequisite for using boundary element method (BEM) and finite element method (FEM) in the implicit solvent models, and it is a more demanding task than for the only purposes of visualization and/or geometry analyses of molecular structure. So far, due to the highly complex and irregular shape of the molecular surface,

*Corresponding author (email: bzlu@1sec.cc.ac.cn) efficient meshing of molecular surface for large real biomolecule with high quality remains a challenging problem. Actually, it was historically a great impediment to use $\mathrm{BEM} / \mathrm{FEM}$ in continuum modeling of large molecular systems.

The main purpose of this paper is to review the various definitions and meshing methods for molecular surface, including our recent work, and introduce their applications in implicit solvent modeling and simulation. Following this introduction section, in Section 1, various definitions of molecular surfaces and corresponding meshing methods are introduced. In Section 2, our recent algorithm and software, TMSmesh, are focused. We also made comparisons with some other methods. In Section 3, a closely related topic, volume mesh generation, is briefly discussed. In Section 4, we present some numerical results of TMSmesh from preliminary experiments and demonstrate its applicability to continuum modeling of molecular system. Concluding remarks are given in Section 5 . 


\section{Some definitions and meshing methods for molecular surface}

Various definitions of molecular surface exist, including the van der Waals (VDW) surface, solvent accessible surface (SAS) [2], solvent excluded surface (SES) [3], molecular skin surface [4], the minimal molecular surface [5] and Gaussian surface.

The VDW surface is defined as the surface of the union of the spherical atomic surfaces with the VDW radius of each atom in the molecule. The SAS and SES are represented by the trajectory of the center and the interboundary of a rolling probe on the VDW surface, respectively (Figure 1).

The molecular skin surface is the envelope of an infinite family of spheres derived from atoms by convex combination and shrinking. The minimal molecular surface is defined as a result of the surface free energy minimization. Different from these definitions, the Gaussian surface is defined as a level set of the summation of the Gaussian kernel functions as follows:

$$
\left\{\vec{x} \in \mathbb{R}^{3}, \phi(\vec{x})=t_{0}\right\},
$$

where

$$
\phi(\vec{x})=\sum_{i=1}^{N} e^{-d\left(\left\|\vec{x}-\vec{c}_{i}\right\|^{2} / r_{i}^{2}-1\right)},
$$

the parameter $d$ is positive and controls the decay speed of the kernel functions, $\vec{c}_{i}$ and $r_{i}$ are the location and radius of atom $i$, and $t_{0}$ and $d$ are usually set as 1 and 0.5. Compared with other definitions of molecular surface, Gaussian surface is smooth and more suitable to represent the electron density of a molecule [6]. The VDW surface, SAS and SES can be approximated well by the Gaussian surface with proper parameter selection $[6,7]$. The Gaussian surface has been widely used in many problems in computational biology, such as docking problem [8], molecular shape comparison [9], calculating SAS area [10] and the generalized Born models [11].

With various definitions of molecular surface that has been proposed, numerous works have been devoted to the computation of molecular surface.

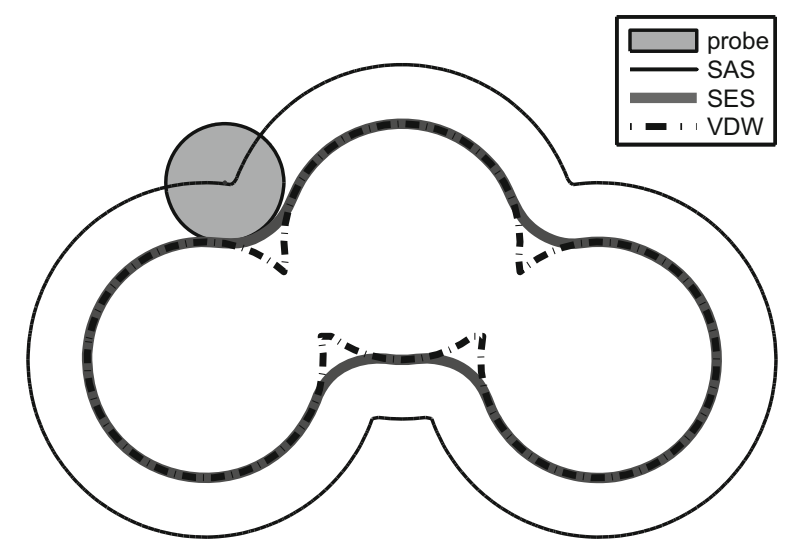

Figure 1 An illustration of VDW, SAS and SES.
For SAS and SES, the representative methods are as follows. Connolly's method is the pioneer work on calculating the molecular surface and SAS analytically $[12,13]$. In Connolly's work, molecular surface is represented by three kinds of patches: convex spherical patches, saddle-shaped toroidal patches, and concave spherical triangular patches. These patches are identified by the number of atoms contacted by the probe. In 1995, a popular program, GRASP, for visualizing molecular surfaces was presented [14]. An algorithm named SMART for triangulating SAS into curvilinear elements was proposed by Zauhar in [15]. Sanner et al. presented a tool, named MSMS, for meshing the SES [16]. MSMS contains four algorithms. The first algorithm is to compute the reduce surface of the atoms. The second algorithm builds the analytical representation of the solvent excluded surface base on the reduce surface produced by the first algorithm. The third algorithm handles the singularities created by the second algorithm. The last one triangulates the solvent excluded surface. MSMS is one of the most widely used softwares for molecular surface triangulation because of its high efficiency. In 1997, Vorobjev et al. [17] proposed SIMS, a method of calculating a smooth invariant molecular dot surface, in which an exact method for removing selfintersecting parts and smoothing the singular regions of the SES was presented. Ryu et al. [18] proposed a method based on Beta-shapes, which is a generalization of $\alpha$ shapes [19]. Can et al. [20] proposed LSMS to generate the SES on grid points using level-set methods. In LSMS, the molecular surface is reached by propagating an initial seed surface using fast marching method [21].

For Skin surface, Chavent et al. [22] presented MetaMtal to visualize the molecular skin surface. Different from existing software that discretizes the surface with triangles or grids, MetaMtal directly visualizes pixels on the molecular skin using a GPU accelerated ray-casting method. In the skin surface meshing method proposed by Cheng et al. [23], a quality molecular skin surface mesh is generated from Delaunay triangulation of the sample points on the skin surface.

For minimal molecular surface, Wei et al. [5] constructed a surface-based energy functional, and use minimization and isosurface extraction processes to obtain a so-called minimal molecular surface. But meshing method was not focused in their work.

For the Gaussian surface, existing techniques for triangulating an implicit surface can be used to mesh the Gaussian surface. These methods are divided into two main categories: spatial partition and continuation methods. The well known marching cubes method [24] and dual contouring method [25] are examples of the spatial partition methods. In these methods, 3D space is divided into cells and an underlying continuous function, typically derived from a spatial density, is assumed to define the surface. Polygonal faces approximating the surface are generated at appropriate cells by examining the signature of the signs of the function at the corners of each cell. It is supposed that the underlying function is 
approximately linear in each cell. In 2006, Zhang et al. [26] used a modified dual contouring method to generate meshes for biomolecular structures. A later tool, GAMer [27, 28], was developed for improving the qualities of the meshes of Gaussian surface produced by marching cubes method.

The continuation methods [29-31] are of another category. These methods mesh the implicit surface by growing current polygonization's border through adding new vertices. The quality of mesh triangles is well controlled in these meshing processes, but techniques for avoiding overlapping, filling the gap between adjacent branches and selecting proper initial triangles are required.

Software packages based on these surface definitions and meshing methods generate acceptable meshes for visualization purposes, but lead to differences in the calculation of free energy and electrostatic properties. Also, some of them display poor performance in efficiency or numerical stability. For example, we observed that MSMS, although highly efficient, generated from time to time non-manifolded meshes with isolated nodes and intersecting triangles, and even failed for large size molecules. Here, a surface is a manifold means each point on the surface has a neighborhood homeomorphic to a disk in the real plane. Meshing a manifold surface should also produce a manifold mesh, and a manifold mesh means that the surface formed by all the elements of the mesh is also a manifold. A low quality non-manifold mesh may significantly slow down or even inhibit the convergence of BEM/FEM calculations.

\section{A robust manifold meshing method: TMSmesh}

TMSmesh is a method for triangular meshing of the Gaussian surface $[32,33]$. The trace technique which is a generalization of predictor-corrector technique is used in TMSmesh to connect sampled surface points. In TMSmesh, there are no problems of overlapping, gap filling, and selecting initial seeds that need to be considered in traditional continuation methods, because the Gaussian surface is polygonized by connecting presampled surface points. TMSmesh is capable of meshing Gaussian surface for biomolecules consisting of more than one million atoms. The produced meshes of TMSmesh are manifold meshes and applicable to BEM/FEM simulations of bimolecular electrostatics.

The main points of TMSmesh are briefly reviewed as follows. The technical details of TMSmesh can be referred to [32] and [33].

TMSmesh contains two main steps. The first step is to compute the intersecting points between the molecular Gaussian surface and the lines parallel to $x$-axis. In this step, the molecule is placed in a three-dimensional orthogonal grid. The boxes having no surface points are ruled out using the estimated bounds of the implicit function $\phi(x)$ in each box. In the remaining cubes, the intersect points between the sur- face and the lines parallel to $x$-axis are found through root finding algorithms.

In the second step, the sampled surface points are connected through three algorithms to form loops, and the whole closed manifold surface is decomposed into a collection of patches enclosed by loops on the surface. The first is the trace step which connects two adjacent topologically connected surface points on the lines parallel to $x$-axis. In the trace step, the predictor-corrector method is used to generate the next corrected surface point from the current one, and the topology connection is confirmed by checking the continuity between the corrected and the current points. If the continuity is not fulfilled, the predictor-corrector is restarted from the current point with a smaller step size. During this trace process, some extreme point along $\alpha$ direction $(\alpha \in\{x, y, z\})$ in the surface, i.e. the point $\vec{x}_{0}$ satisfying $\left.\frac{\partial \phi(\vec{x})}{\partial \alpha}\right|_{\vec{x}=\vec{x}_{0}}=0$ and $\phi\left(\vec{x}_{0}\right)=t_{0}$, is also found by checking the sign change of $\frac{\partial \phi(\vec{x})}{\partial \alpha}$ on the traced path.

The second algorithm gives the sequence of the trace steps on $x y$ and $x z$ planes to ensure the connected surface points on lines parallel to $x$-axis forming loops on the surface. With these two algorithms, all the sampled surface points are connected and formed close loops on surface and the whole surface is divided in to patches enclosed by these loops. Figure 2 illustrates a process of connecting the sampled surface points to form close loops.

These patches are often non-single valued along at least one of $x, y, z$ directions, and may contain holes and tunnels. In the step of triangulating the non-single valued patches, these holes or tunnels may be missed, and intersections may occur. This causes difficulties in guaranteeing a triangulated manifold and preserving the surface topology, even with very small grid spaces. To avoid these difficulties, it is necessary to dissect each patch enclosed by loop into single valued pieces in $x, y, z$ directions further, the following third algorithm is employed in TMSmesh.

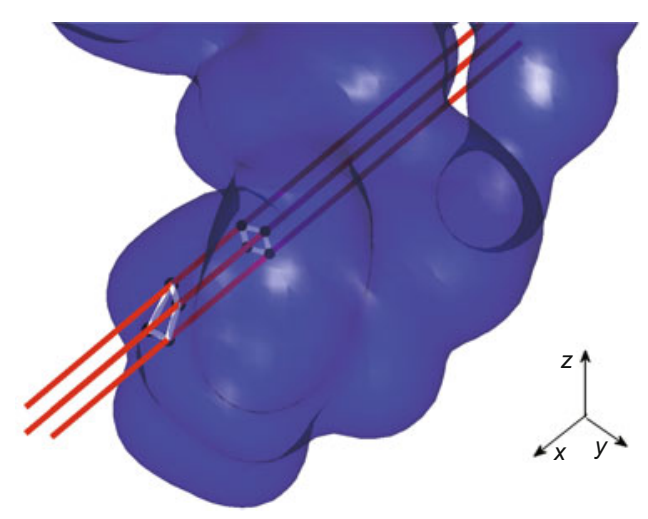

Figure 2 An illustration of a process of connecting the sampled surface points to form close loops. The Gaussian molecular surface is in blue color. Black points are intersecting points between the surface and the lines parallel to $x$-axis. The white lines connected the intersect points form polygons on the surface. 
In the third algorithm, the extreme points along the fold curves are also connected using the trace algorithm. The fold curves for $x, y, z$ directions are defined as follows:

$$
\left\{x \in \mathbb{R}^{3}, \phi(x)=t_{0}, \frac{\partial \phi(x)}{\partial \alpha}=0\right\}, \alpha \in\{x, y, z\}
$$

Cutting the Gaussian surface defined in eq. (1) along fold curves ensures the resulted pieces are single valued on $x, y, z$ directions. Then, these pieces can be treated as 2-dimensional polygon and be easily triangulated through standard triangulation algorithms [34]. The third algorithm ensures the resulted meshes are manifolds without intersections and preserve the topology of the surface. Figure 3 shows an example that the fold curves dividing a surface patch into single valued pieces on $x, y, z$ directions.

Most of existing molecular surface meshing methods, such as GRASP, MSMS, and LSMS were designed for molecular visualization and geometry analysis in computational structure biology or structural bioinformatics. However, for advanced mathematical modeling in the field of implicit solvent modeling using FEM/BEM, qualities of the produced meshes usually need to be improved through carefully checking and rearranging (eliminating the intersections between elements, picking out the irregular nodes/edges/elements and rearranging the mesh) to make the meshes be manifold mesh. And manifoldness is one of the primary requirements of the surface mesh that can be used in FEM/BEM computations.

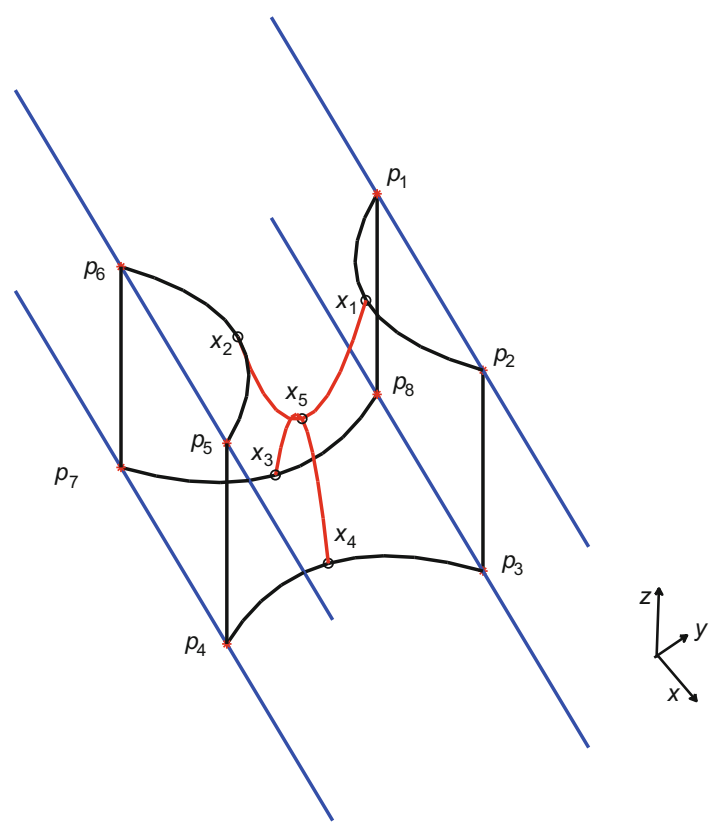

Figure 3 An example of connecting surface extreme points along the fold curves using the third algorithm in TMSmesh. The red curves are fold curves. $p_{i}, i=1, \cdots, 8$ are the intersection points between the Gaussian surface and the lines parallel to $x$-axis. The black curves form a close loop on the surface. The surface patch enclosed by the black loop is not single valued along $x, y$ directions and it is divided into four single valued pieces along $x, y$ directions by the fold curves. They are $x_{1}-p_{2}-p_{3}-x_{4}-x_{5}$, $p_{1}-x_{1}-x_{5}-x_{3}-p_{8}, x_{3}-x_{5}-x_{2}-p_{6}-p_{7}, x_{5}-x_{4}-p_{4}-p_{5}-x_{2}$.
In [32] and [33], the performance of TMSmesh is shown to be improved in the following aspects compared with MSMS. Firstly, TMSmesh is robust. For our benchmark set consisting of molecules with the number of atoms ranged from hundreds to one millions, TMSmesh successfully produces all the surface meshes. Secondly, the meshes produced by TMSmesh have good qualities. To verify this, the histograms of ratios of the longest edge length to the shortest length of each triangle of the meshes show that TMSmesh produces less sharp triangles. Also, it is found that TMSmesh has smoother convergent behaviors of areas and volumes of surface meshes than MSMS when the grid space tends to zero. Furthermore, as presented in [33], TMSmesh generates manifold meshes and preserved the topology of molecular surface. However, as to the aspect of computational efficiency, although the computational complexity is linear with respect to the number of atoms as showed in [32], the efficiency of TMSmesh still needs to be improved.

In [32], we also compare TMSmesh with LSMS that is a fast meshing and visualization software for molecular surface using level-set method. TMSmesh is different with LSMS in the following aspects. Firstly, LSMS visualizes the molecular surface based on cubic grids. Therefore, the surfaces produced by LSMS deviate to certain considerable degree from the molecular surface (unless the grid space is small enough). Secondly, LSMS uses level-set method which evolves a surface wrapped the molecular surface. It has a computational complexity of $O\left(L^{3}\right)$, where $L$ is the number of grids in one dimension. Therefore, the memory requirement increases dramatically when $L$ becomes large. For our benchmark set, LSMS failed for two large molecules consisting of more than half million atoms due to too large memory requirements. For TMSmesh, the computational complexity is $O(N)$, where $N$ is the number of atoms. The numerical tests show that TMSmesh costs less memory, but more CPU time than LSMS.

Differing from another meshing tool for Gaussian surface, GAMer [28], which also employs marching cubes method to generate the initial surface mesh followed by using an anglebased quality improvement algorithm to improve the surface mesh qualities, TMSmesh generates the surface mesh directly by several algorithms based on techniques of root finding, predictor-corrector and tracing fold curves. With these techniques, TMSmesh now can treat arbitrarily large biomolecule in PDB, and the generated surface mesh is guaranteed to be manifold mesh, and also importantly the surface mesh is faithful to the original molecular surface geometry.

\section{Volume mesh generation}

The molecular surface mesh can be used for generation of the corresponding surface conforming volume mesh. A tool chain for high-quality biomolecule volume mesh generation can be built by using a number of existing mesh generation 
tools. The tool chain has essentially three components: surface meshing, quality improving, and volume mesh generation. First, triangular surface mesh can be generated using the program TMSmesh or other tools. Second, the surface mesh can be further treated or improved. For example, the program ISO2Mesh [35] can be used to simplify the surface mesh by reducing the number of faces or adding some points to the surface mesh while keeping manifoldness, and persevering the overall shape, volume and boundaries as much as possible. Finally, in the third step, the tetrahedral volume mesh is generated using a third party program such as TetGen [36]. A set of switches are used to control the behavior of TetGen. In general, we use the switch command -pq to get high-quality tetrahedral mesh. The $-\mathrm{p}$ switch reads a piecewise linear complex (PLC) stored in file .poly or .smesh and generates a constrained Delaunay tetrahedralization of the PLC. The -q switch performs quality volume mesh generation by Shewchuks Delaunay refinement algorithm [37].

\section{Application of molecular surface mesh to BEM and FEM in continuum modeling of molecular systems}

The molecular surface mesh generated by many current mesh generation softwares can be applied to molecular visualization and analysis of surface area, topology, and volume in computational structure biology and structural bioinformatics. Furthermore, molecular surface mesh can be applied to some advanced mathematical modeling of biomolecules, which places demands upon the quality and the rigorous topology of the surface and volume meshes. In our previous work [32], we have already shown that the mesh generated by TMSmesh can be successfully applied to BEM calculations with better convergence performance and lead to reasonable results. The volume tetrahedral mesh generated from the TMSmesh surface mesh by the tool chain described in this review also show good performance in the usage of FEM computations [38].

Here, we used a relatively large molecule to show the applications of molecular surface mesh to FEM computation. The protein is a protongated ion channel from Gloeobacter violaceus (GLIC, PDB code: 3ehz), and the structure is taken from a MD simulation trajectory [39]. GLIC consists of 25300 atoms, in $100 \AA \times 80 \AA \times 128 \AA$ dimensions. In our experiments, the molecular surface meshes generated by MSMS, with $1 / \AA^{2}$ density and either 1.5 or $1.4 \AA$ of probe radius, contained many isolated nodes. Such topological faults inhibited successful volume mesh generation. In comparison, the molecular surface mesh generated by the TMSmesh, with 329764 triangular elements and 164523 nodes (Figure 4), allowed successful volume mesh generation and FEM calculation.

Figure 4 shows an example of an unstructured tetrahedral volume mesh and a triangulated surface mesh of GLIC by using a meshing tool chain as described in Section 3.

Figure 5 shows the FEM results of Poisson-Boltzmann electrostatic potential of GLIC. A very smooth numerical solution is obtained over the molecular surface.

\section{Conclusion}

Various molecular surface definitions and surface meshing methods have been reviewed in this paper. In particular, the focus is put on a challenging and urgent issue - efficient and quality mesh generation of large molecular surface, because it is required for a range of emerging approaches in mathematical modeling of biomolecular systems.

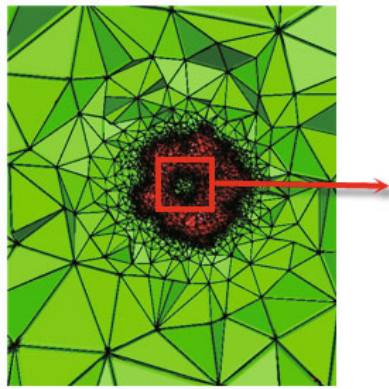

(a)

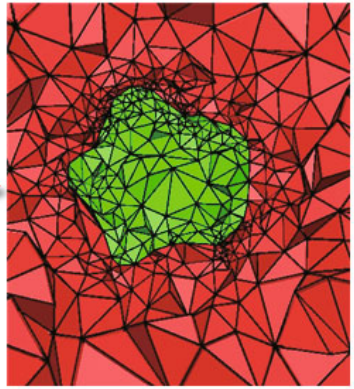

(b)

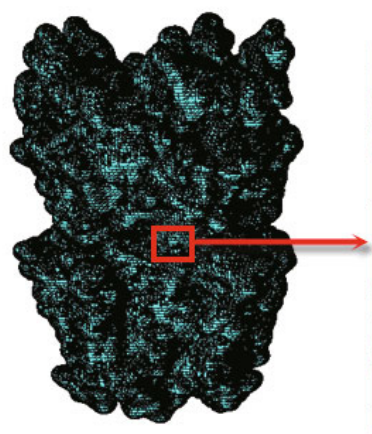

(c)

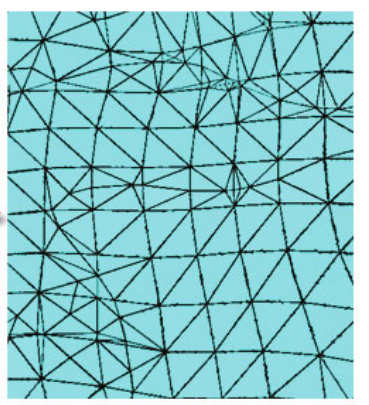

(d)
Figure 4 An example of mesh generation for GLIC. (a) Cross section of the whole tetrahedral volume mesh; (b) a close-up view of the fine mesh around the molecule, whose body is colored by red; (c) the triangular boundary mesh conforming to the molecular surface; (d) a close-up view of the molecular surface mesh.

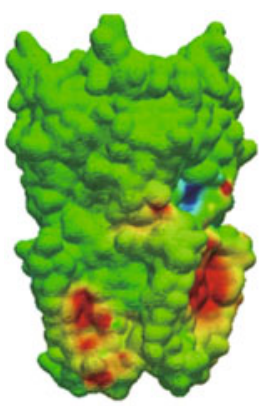

(a)

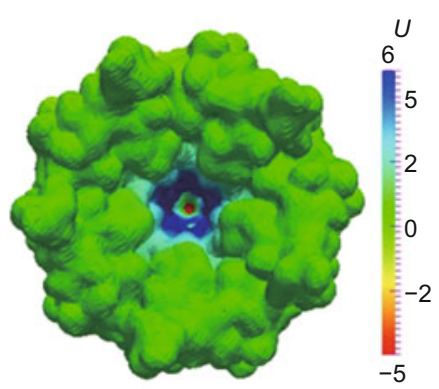

(b)
Figure 5 Surface electrostatic potential of GLIC. (a) A side view of surface electrostatic potential from the Poisson-Boltzmann solution. The color scale is from -5 (red) to 6 (blue) $\mathrm{kcal} / \mathrm{mol} \cdot \mathrm{e}$. (b) A top view of the surface electrostatic potential. 
Among these methods, TMSmesh is detailed in this review, and it is shown as an effective and robust way to get high-quality Gaussian molecular surface mesh in the sense that: (1) Arbitrary-sized molecule available in PDB can be stably handled by TMSmesh on a typical desktop or laptop machine, even for the not "good" molecular structures (such as ones with strong atomic clashes), (2) TMSmesh produces meshes with good quality (manifoldness, uniformness), and (3) the generated surface mesh preserves the original molecular surface features and properties (topology, surface area and enclosed volume, and local curvature). The meshes are also shown to be successfully applicable to numerical simulations with BEM/FEM. Furthermore, the meshing method developed in TMSmesh can be generalized for meshing different types of implicit surfaces emerging in other fields, such as computer graphics, medical imaging, geometry modeling and structural optimization and simulation [40,41].

In order to simulate more complicated and wider ranges of biophysical processes using a variety of numerical techniques and modeling approaches, the current meshing methods need improvements. Firstly, the surface mesh quality needs to be further improved, as it influences the generation and quality of volume mesh, and the mesh quality closely relates to the condition number of the generated linear system in BEM/FEM, and consequently influences the performances of their numerical computations. Secondly, the efficiency seems to be the current bottleneck in some possible applications where the mesh needs to be either generated for large systems or generated frequently, such as in multipleconformational analysis, BEM or FEM-based implicit solvent MD simulations [42,43].

Finally, for implicit solvent modeling, which surface definition is the best is still hard to concluded, as some other factors (like the atomic radii) in the setup of a implicit solvent calculation can also affect the final results. In the future, different definitions, including the new definitions based on nonadditivity relation [44] or more generalized entropy form [45], and the meshing approaches will need systematic studies and comparisons with experiments or other computational methods.

We thank B. Tu for his graphic work in this manuscript. M.X. Chen was supported by the Collegiate Natural Science Foundation of Jiangsu Province (11KJB110010) and National Natural Science Foundation of China (91230106, 11001062). B.Z. Lu was supported by the State Key Laboratory of Scientific/Engineering Computing, the National Center for Mathematics and Interdisciplinary Sciences, Chinese Academy of Sciences, National High-Tech Research and Development Program of China (2012AA020403) and National Natural Science Foundation of China (10971218, 91230106).

1 Zhu D, Liao X, Dai P. Theoretical analysis of reactive solid-liquid interfacial energies. Chin Sci Bull, 2012, 57: 4517-4524

2 Lee B, Richards F M. The interpretation of protein structures: Estimation of static accessibility. J Mol Biol, 1971, 55: 379-384

3 Richards F M. Areas, volumes, packing and protein structure. Ann Rev Biophys Bioeng, 1977, 6: 151-176

4 Edelsbrunner H. Deformable smooth surface design. Discrete Comput
Geom, 1999, 21: 87-115

5 Bates P W, Wei G W, Zhao S. Minimal molecular surfaces and their applications. J Comput Chem, 2008, 29: 380-391

6 Duncan B S, Olson A J. Shape analysis of molecular surfaces. Biopolymers, 1993, 33: 231-238

7 Blinn J F. A generalization of algebraic surface drawing. ACM Trans Graph, 1982, 1: 235-256

8 Mcgann M R, Almond H R, Nicholls A, et al. Gaussian docking functions. Biopolymers, 2003, 68: 76-90

9 Grant J A, Gallardo M A, Pickup B T. A fast method of molecular shape comparison: A simple application of a Gaussian description of molecular shape. J Comput Chem, 1996, 17: 1653-1666

10 Weiser J, Shenkin P, Still W. Optimization of gaussian surface calculations and extension to solvent-accessible surface areas. J Comput Chem, 1999, 20: 688-703

11 Yun Z, Matthew P, Richard A. What role do surfaces play in GB models? A new-generation of surface-generalized born model based on a novel gaussian surface for biomolecules. J Comput Chem, 2005, 27: 72-89

12 Connolly M L. Analytical molecular surface calculation. J Appl Crystallogr, 1983, 16: 548-558

13 Connolly M L. Solvent-accessible surfaces of proteins and nucleic acids. Science, 1983, 221: 709-713

14 Nicholls A, Bharadwaj R, Honig B. Grasp: Graphical representation and analysis of surface properties. Biophys J, 1995, 64: 166-167

15 Zauhar R J. Smart: A solvent-accessible triangulated surface generator for molecular graphics and boundary element applications. J Comput Aid Mol Des, 1995, 9: 149-159

16 Sanner M, Olson A, Spehner J. Reduced surface: An efficient way to compute molecular surfaces. Biopolymers, 1996, 38: 305-320

17 Vorobjev Y N, Hermans J. SIMS: Computation of a smooth invariant molecular surface. Biophys J, 1997, 73: 722-732

18 Ryu J, Park R, Kim D S. Molecular surfaces on proteins via beta shapes. Comput Aided Design, 2007, 39: 1042-1057

19 Edelsbrunner H, Mucke E P. Three-dimensional alpha shapes. ACM Trans Graph, 1994, 13: 43-72

20 Can T, Chen C I, Wang Y F. Efficient molecular surface generation using level-set methods. J Mol Graph Model, 2006, 25: 442-454

21 Sethian J A. Level Set Methods and Fast Marching Methods: Evolving Interfaces in Computational Geometry, Fluid Mechanics, Computer Vision, and Materials Science. 2nd ed. London: Cambridge University Press, 1999

22 Chavent M, Levy B, Maigret B. Metamol: High-quality visualization of molecular skin surface. J Mol Graph Model, 2008, 27: 209-216

23 Cheng H, Shi X. Quality mesh generation for molecular skin surfaces using restricted union of balls. Comput Geom, 2009, 42: 196-206

24 Lorensen W, Cline H E. Marching cubes: A high resolution 3d surface construction algorithm. Comput Graph, 1987, 21: 163-169

$25 \mathrm{Ju}$ T, Losasso F, Schaefer S, et al. Dual contouring of hermite data. ACM Trans Graph, 2002, 21: 339-346

26 Zhang Y, Xu G X, Bajaj R. Quality meshing of implicit solvation models of biomolecular structures. Comput Aided Geom Des, 2006, 23: 510-530

27 Yu Z, Holst M J, McCammon J A. High-fidelity geometric modeling for biomedical applications. Finite Elem Anal Des, 2008, 44: 715-723

28 Yu Z, Holst M J, Yuhui C, et al. Feature-preserving adaptive mesh generation for molecular shape modeling and simulation. J Mol Graph Model, 2008, 26: 1370-1380

29 Hilton A, Stoddart A J, Illingworth J, et al. Marching triangles: Range image fusion for complex object modelling. IEEE Int Conf Image Proc, 1996, 1: 381-384

30 Hartmann E. A marching method for the triangulation of surfaces. Vis 
Comput, 1998, 14: 95-108

31 Karkanis T, Stewart J. Curvature dependent triangulation of implicit surfaces. IEEE Comput Graph Appl, 2001, 21: 60-69

32 Chen M X, Lu B Z. Tmsmesh: A robust method for molecular surface mesh generation using a trace technique. J Chem Theory Comput, 2011, 7: 203-212

33 Chen M X, Tu B, Lu B Z. Triangulated manifold meshing method preserving molecular surface topology. J Mol Graph Model, 2012, 38 : 411-418

34 de Berg M, van Kreveld M, Overmars M, et al. Computational Geometry: Algorithms and Applications. 2nd ed. New York: Springer-Verlag, 2000

35 Iso2mesh: A free $3 \mathrm{~d}$ surface and volumetric mesh generator for matlab/octave. http://iso2mesh.sourceforge.net

36 Si H, Gaertner K. Meshing piecewise linear complexes by constrained delaunay tetrahedralizations. In: Proceedings of the 14th International Meshing Roundtable, 2005. 147-163

37 Shewchuk J R. Tetrahedral mesh generation by delaunay refinement. In: Proceedings of the 14th Annual ACM Symposium of Computational Geometry, 1998. 86-95

38 Tu B, Chen M X, Xie Y, et al. A parallel finite element simulator for ion transport through three-dimensional ion channel systems. J Comput Chem, 2013, in press

39 Fritsch S, Ivanov I, Wang H L, et al. Ion selectivity mechanism in a bacterial pentameric ligand-gated ion channel. Biophys J, 2011, 100: 390-398

40 Jiang H W, Wu S T, Cheng J S. Optimization model of a structural simulation design for a CICC. Chin Sci Bull, 2011, 56: 2978-2983

41 Gao T, Cui K, Wang X, et al. Aerodynamic optimization and evaluation for the three-dimensional afterbody/nozzle integrated configuration of hypersonic vehicles. Chin Sci Bull, 2012, 57: 849-857

42 Wang C X, Wan S Z, Xiang Z X, et al. Incorporating hydration force determined by boundary element method into stochastic dynamics. J Phys Chem B, 1997, 101: 230-235

43 Chen W Z, Lu B Z, Wang C X. A simulation method of combining boundary element method with generalized langevin dynamics. Chin Sci Bull, 2000, 45: 2227-2231

44 Wang R, Djanteng S N, Kaabouchi A E, et al. Investigation of an energy nonadditivity for nonextensive systems. Chin Sci Bull, 2011, 56: 3661-3665

45 Ou C J, Chen J C. Generalized entropies under different probability normalization conditions. Chin Sci Bull, 2011, 56: 3649-3653

Open Access This article is distributed under the terms of the Creative Commons Attribution License which permits any use, distribution, and reproduction in any medium, provided the original author(s) and source are credited. 